\title{
Wnt activation downregulates olfactomedin-1 in Fallopian tubal epithelial cells: a microenvironment predisposed to tubal ectopic pregnancy
}

\author{
Suranga P Kodithuwakku ${ }^{1,5}$, Ronald TK Pang ${ }^{1}$, Ernest HY Ng ${ }^{1,2}$, Annie NY Cheung ${ }^{1,3}$, Andrew W Horne ${ }^{4}$, \\ Pak-Chung $\mathrm{Ho}^{1,2}$, William SB Yeung ${ }^{1,2}$ and Kai-Fai Lee ${ }^{1,2}$
}

Ectopic pregnancy (EP) occurs when the embryo fails to transit to the uterus and attach to the luminal epithelium of the Fallopian tube (FT). Tubal EP is a common gynecological emergency and more than $95 \%$ of EP occurs in the ampullary region of the FT. In humans, Wnt activation and downregulation of olfactomedin-1 (Olfm-1) occur in the receptive endometrium and coincided with embryo implantation in vivo. Whether similar molecular changes happen in the FT leading to EP remains unclear. We hypothesized that activation of Wnt signaling downregulates Olfm-1 expression predisposes to EP. We investigated the spatiotemporal expression of Olfm-1 in FT from non-pregnant women and women with EP, and used a novel trophoblastic spheroid (embryo surrogate)-FT epithelial cell co-culture model (JAr and OE-E6/E7 cells) to study the role of Olfm-1 on spheroid attachment. Olfm-1 mRNA expression in the ampullary region of non-pregnant FT was higher $(P<0.05)$ in the follicular phase than in the luteal phase. Ampullary tubal Olfm-1 expression was lower in FT from women with EP compared to normal controls at the luteal phase (histological scoring $(\mathrm{H}-\mathrm{SCORE})=1.3 \pm 0.2$ vs $2.4 \pm 0.5 ; P<0.05)$. Treatment of OE-E6/E7 with recombinant Olfm- $1(0.2-5 \mu \mathrm{g} / \mathrm{ml})$ suppressed spheroid attachment to OE-E6/E7 cells, while activation of Wnt-signaling pathway by Wnt3a or LiCl reduced endogenous Olfm-1 expression and increased spheroid attachment. Conversely, suppression of Olfm-1 expression by RNAi increased spheroid attachment to OE-E6/E7 cells. Taken together, Wnt activation suppresses Olfm-1 expression, and this may predispose a favorable microenvironment of the retained embryo in the FT, leading to EP in humans.

Laboratory Investigation (2012) 92, 256-264; doi:10.1038/labinvest.2011.148; published online 3 October 2011

KEYWORDS: Fallopian tube; olfactomedin-1; tubal ectopic pregnancy; Wnt signaling

Ectopic pregnancy occurs when an embryo implants outside the uterus. It happens in $1-2 \%$ of pregnancies and is associated with significant morbidity and mortality. Interestingly, about $85-95 \%$ of the ectopic pregnancies occur in the ampullary region (distal 2/3) of the Fallopian tube. ${ }^{1}$ Genital pelvic infection and cigarette smoking are the risk factors associated with tubal ectopic pregnancy (TEP). ${ }^{2}$ Moreover, changes in extracellular matrix molecules, cytokines and other growth factor expressions are also reported in the Fallopian tube of patients with TEP. Yet, the etiology of TEP is still not fully understood.
The olfactomedins (Olfms) are a family of secretary glycoproteins first identified in the olfactory epithelial tissues of the Xenopus. ${ }^{3}$ To date, over 50 species, including rats, frogs, sea urchins, cats, dogs, zebra fish and humans, are found to express Olfms. ${ }^{4}$ All the well-characterized Olfm proteins share a common Olfm domain of about 250 amino acids in size at their $\mathrm{C}$ termini. ${ }^{4}$ In mammals, there are about 13 wellcharacterized Olfm domain-containing proteins ${ }^{4}$ and among the best understood Olfms; Olfm-1, -2, -3, -4 and myocilin are known to regulate cellular growth, differentiation and pathological processes.

\footnotetext{
'Department of Obstetrics and Gynaecology, Li Ka Shing Faculty of Medicine, The University of Hong Kong, Pokfulam, Hong Kong, China; ${ }^{2}$ Center for Reproduction, Development and Growth, Li Ka Shing Faculty of Medicine, The University of Hong Kong, Pokfulam, Hong Kong, China; ${ }^{3}$ Department of Pathology, Li Ka Shing Faculty of Medicine, The University of Hong Kong, Pokfulam, Hong Kong, China; ${ }^{4}$ Center for Reproductive Biology, Queen's Medical Research Institute, University of Edinburgh, Edinburgh, UK and ${ }^{5}$ Department of Animal Science, The University of Peradeniya, Peradeniya, Sri Lanka

Correspondence: Dr K-F Lee, PhD, Department of Obstetrics and Gynaecology, Li Ka Shing Faculty of Medicine, The University of Hong Kong, Pokfulam, Hong Kong, China.
}

E-mail: ckflee@hku.hk

Received 24 June 2011; revised 9 August 2011; accepted 11 August 2011 
Olfm- 1 is one of the most studied members in the family. The Olfm-1 gene is located on chromosome 9q34.3. Four variant transcripts are produced by alternative splicing. ${ }^{5}$ The longest transcript encodes a protein of 464 amino acids with a native molecular weight of $57 \mathrm{kDa}$. Olfm-1 is expressed in the human brain, eye, liver, lung, heart, as well as uterine tissues. The exact function of Olfm-1 is not known, but proteins containing an Olfm-like domain may play important roles on cell adhesion ${ }^{6}$ or act as scaffolding proteins. ${ }^{7,8}$ Recently, Olfm-1 was found to modulate the canonical Wnt-signaling pathway, which regulates various cellular functions. ${ }^{9}$

Recent microarray data have shown that the Olfm-1 transcript is downregulated in the endometrium during the window of implantation, ${ }^{10-13}$ and that upregulation of Olfm-1 transcript is associated with pathological conditions, including endometriosis ${ }^{14}$ and recurrent spontaneous abortions. ${ }^{14,15}$ Recently, we reported that Olfm-1 expression in the endometrial epithelium is lower at the luteal phase than at the follicular phase of the menstrual cycle, suggesting that progesterone may suppress Olfm-1 expression. ${ }^{16}$ In addition, using a trophoblastic spheroid (JAr)-endometrial epithelial cell ${ }^{17}$ co-culture model, we have shown that Olfm-1 dose-dependently downregulates JAr spheroid attachment onto Ishikawa cells. ${ }^{16}$

We hypothesized that aberrant expression of Olfm-1 in the Fallopian tube may be one of the predisposing factors leading to ectopic pregnancy in humans. Olfm-1 interacts with Wntsignaling inhibitor (WIF-1), ${ }^{9}$ and therefore modulates Wntregulated genes important for embryo implantation and early development. ${ }^{18,19}$ In this study, we compared the spatiotemporal expression of Olfm-1 in Fallopian tube from nonpregnant women and from women with ectopic pregnancies, and used a novel trophoblast spheroids (embryo surrogate)Fallopian tubal epithelial cell co-culture model (JAr and OEE6/E7 cells) to study the role of Olfm-1 in regulating Wnt signaling and tubal-embryo attachment.

\section{MATERIALS AND METHODS Patient Samples}

All non-pregnant Fallopian tubes (ipsilateral and contralateral side) were obtained from 29 patients (age: $36-51$ years; mean \pm s.d.: $45 \pm 4.9$ years) who had undergone hysterectomy, had regular menstrual cycle (28-30 days) and no known tubal pathological conditions with written consent. The phase of menstrual cycle was determined by the date of the last menstrual period. Fallopian tubes with implantation site at the ampullary region were retrieved from our archive samples from 10 patients (age: $26-42$ years; mean \pm s.d.: $33 \pm 5.2$ years; gestational age: $7-10$ weeks) who had undergone salpingectomy for the treatment of TEP. The study was approved by the Institutional Review Board of the University of Hong Kong/Hospital Authority Hong Kong West Cluster (UW10-109).

\section{Quantitative PCR and Analysis}

The Fallopian tube from the non-pregnant women who had hysterectomy for non-tubal pathological conditions was dissected longitudinally, and the mucosal layers of different regions (ampulla, infundibulum and isthmus) were carefully peeled off using a forcep as described elsewhere. ${ }^{20}$ Total RNA was isolated using the Absolutely RNA RT-PCR Miniprep Kit (Stratagene, La Jolla, CA, USA). The integrity of total RNA was determined by gel electrophoresis and spectrophotometry. Then, $400 \mathrm{ng}$ of total RNA was reverse transcribed to synthesize cDNA using the TaqMan RT Reagents (N8080234; ABI Biosystems, Foster City, CA, USA). Human Olfm-1, -2, -3 and -4 transcript-specific TaqMan probes (Supplementary Table 1) were used with the ABI 7500 for quantitative analysis of Olfm transcripts. All qPCR assays were performed with TaqMan PCR Master Mix (Applied Biosystems) and a standard PCR cycling protocol. Human $18 \mathrm{~S}$ ribosomal RNA was used as the internal control to normalize the Olfm expression in the samples. The relative mRNA expression was quantified using $2^{-\Delta \Delta c t}$ method as described elsewhere. ${ }^{21}$ The qPCR experiment was repeated three times in duplicates under similar conditions.

\section{Immunohistochemistry and Histological Scoring}

Fallopian tube biopsies obtained from non-pregnant women and from women with ectopic pregnancies were fixed with $4 \%$ paraformaldehyde, embedded in paraffin wax and sectioned at $5 \mu \mathrm{m}$ for immunohistochemistry (IHC) studies as described previously. ${ }^{16}$ Both the non-pregnant and ectopic pregnancy Fallopian tubes were subjected to IHC using polyclonal anti-Olfm-1 antibody (1:100), which bind specifically to the long form of the Olfm-1 protein. ${ }^{16}$ To confirm the presence of an intact epithelium in the ectopic pregnancy sections, cytokeratin (1:100; DakoCytomation, Glostrup, Denmark) staining was also performed. DAB Substrate Chromogen (DakoCytomation) was used to detect positive staining. Images were captured using a digital camera mounted on a light microscope (Axioscop, Zeiss, Göttingen, Germany). The intensity of Olfm- 1 staining in the Fallopian tube epithelium in a total of 500 cells (five fields with 100 cells each) in each section (2-4 sections each) was subjected to histological scoring (H-SCORE) analysis by two independent observers as described previously. ${ }^{22}$ Results are presented as mean \pm s.d.

\section{Western Blotting}

OE-E6/E7 cells were lysed in RIPA buffer $(1 \mathrm{ml})$ supplemented with protease inhibitors as described. ${ }^{23}$ Protein loading was normalized using the total protein concentrations as determined by the Bradford assay. Antibodies against Olfm-1 (1:1000; Zymed, San Francisco, CA, USA), $\beta$-catenin (1:2500, Cat. No.: 610153; BD Transduction Laboratory, Franklin Lakes, NJ, USA), active- $\beta$-catenin (1:1000, Cat. No.: 05-665; Millipore, Temecula, CA, USA), GSK3- $\beta$ (1:1000, Cat. No.: 610201; BD Transduction Laboratory), E-cadherin 
(1:1000, Cat. No.:1b1416; Abcam, Cambridge, MA, USA) and $\beta$-actin (1:10 000, Cat. No.: A3854; Sigma-Aldrich, St Louis, MO, USA) were used as primary antibodies, whereas horseradish peroxidase-conjugated goat anti-rabbit IgG and sheep anti-mouse IgG (1:5000; GE Healthcare, Piscataway, NJ, USA) were used as secondary antibodies. Specific signal was visualized by the enhanced chemiluminescence method.

\section{Trophoblastic Spheroid (Embryo Surrogate)-Fallopian Tubal Epithelial Cell Co-Culture Model}

A previously established co-culture model to simulate trophoblast-endometrium attachment was modified. ${ }^{16,23} \mathrm{~A}$ human trophoblastic choriocarcinoma cell line (JAr, HTB144; ATCC, Manassas, VA, USA) and human immortalized Fallopian tubal epithelial cell line $(\mathrm{OE}-\mathrm{E} 6 / \mathrm{E} 7)^{24}$ were cultured at $37^{\circ} \mathrm{C}$ in a humid atmosphere with $5 \% \mathrm{CO}_{2}$ in air. The JAr cells were maintained in RPMI 1640 (Sigma), supplemented with 10\% fetal bovine serum (FBS; Invitrogen, Carlsbad, CA, USA), $2 \mathrm{mM}$ L-glutamine and penicillin/streptomycin (100 U/ml and $0.1 \mathrm{mg} / \mathrm{ml}$; Gibco, Carlsbad, CA, USA). Dulbecco's modified essential medium/F12 (DMEM/F12) supplemented with $10 \%$ FBS, L-glutamine and penicillin/ streptomycin were used for culturing the OE-E6/E7 cells. Briefly, OE-E6/E7 cells were treated with or without $1 \mu \mathrm{g} / \mathrm{ml}$ bovine serum albumin (Sigma), human recombinant Olfm-1 (rhOlfm-1) $(0.2,1$ and $5 \mu \mathrm{g} / \mathrm{ml})$, trophoblast differentiation agents: $5 \mu \mathrm{M}$ dibutyryl-cAMP (Sigma), $5 \mu \mathrm{M}$ methotrexate (MTX; Sigma) or progesterone receptor antagonist $4 \mu \mathrm{M}$ RU486 (Tocris) for $24 \mathrm{~h}$. Cloning, overexpression and purification of rhOlfm-1 were described previously. ${ }^{16}$ MTX and RU486 were included as negative controls to validate the coculture assay. ${ }^{16,25,26}$ Multi-cellular spheroids of $60-120 \mu \mathrm{m}$ in size were generated by shaking the trypsinized JAr cells at $6 g$ for $24 \mathrm{~h}$. The spheroids were carefully transferred onto the surface of a confluent OE-E6/E7 monolayer and incubated in DMEM/F12 medium with supplements for up to $24 \mathrm{~h}$. Nonadherent spheroids were removed by agitation at low g-force $(15 g)$ for $10 \mathrm{~min}$. The medium was removed and refilled, and the attached spheroids were counted under a light microscope and expressed as percentage of the total number of seeded spheroids (\% adhesion). Photographs of cultures were taken with a Nikon Eclipse TE300 inverted microscope (Nikon, Tokyo, Japan).

\section{Olfm-1 RNAi Knockdown}

Olfm-1 siRNA from Dharmacon (L-012203-00, $20 \mu \mathrm{M}$ ) and Applied Biosystems $(4392420,20 \mu \mathrm{M})$ was mixed at a $1: 1$ ratio and diluted in Opti-MEM medium. Briefly, $8 \times 10^{5}$ cells in $800 \mu \mathrm{l}$ per well were mixed with $200 \mu \mathrm{l}$ of transfection mixture containing Lipofectamine 2000 and siRNA at a final concentration of $10 \mathrm{nM}$. After $6 \mathrm{~h}$ of transfection, the transfected cells were attached in a 12-well plate and the medium was changed to DMEM/F12 with supplement. The cells were $>90 \%$ confluency after overnight culture. To increase the transfection efficiency of OE-E6/E7 cells, the cells were transfected again. The media were changed to Opti-MEM and a mixture of siRNA with Lipofectamine 2000 was added to the attached cells. The medium was changed and the cells were ready for co-culture after $24 \mathrm{~h}$ of incubation. Negative control siRNA (Dharmacon and Applied Biosystems) was transfected using the same protocol. Western blotting was used to check the level of Olfm-1 knockdown in the OE-E6/ E7 cells using the anti-Olfm-1 antibody described above.

\section{Activation of the Wnt-Signaling Pathway}

Wnt3a-conditioned medium (Wnt3a-CM) was obtained from cultured mouse L-Wnt 3A cells (ATCC CRL-2647) stably secreting Wnt3a as described previously, ${ }^{27,28}$ and used to induce the Wnt-signaling pathway in the OE-E6/E7 cells. Briefly, the mouse fibroblast L cells were cultured in DMEM medium supplemented with 10\% FBS, L-glutamine and penicillin/streptomycin until confluent. The CM was collected at $48 \mathrm{~h}$ after confluency and tested for the presence of Wnt3a by western blotting. The conditioned media obtained from normal mouse fibroblast L cells were used as negative control for the spheroid attachment assay. All CM was filter sterilized and stored at $-20{ }^{\circ} \mathrm{C}$ until used. $\mathrm{LiCl}$ (Sigma) stock solution at a concentration of $5 \mathrm{M}$ was prepared, dissolved in distilled water, filter sterilized and stored at room temperature until use. Wnt3a-CM at 1:2 (v:v) and $1: 1$ ratios and $40 \mathrm{mM} \mathrm{LiCl} \mathrm{in} \mathrm{normal} \mathrm{culture} \mathrm{medium} \mathrm{were}$ used for Wnt-signaling activation.

\section{Statistical Analysis}

All results were expressed as means \pm s.d. Statistical comparisons were performed by one-way ANOVA, followed by the Scheffe's or Tukey's test where appropriate. The H-SCORE data were analyzed using Mann-Whitney U-test and Kruskall-Wallis tests for multiple comparisons. A probability of $P<0.05$ was used to indicate a significant difference. A paired $t$-test was used for comparison of $\mathrm{H}$ SCOREs from different observers. The data were analyzed using the statistical software SPSS (v.15.1 for windows; SPPSS, Chicago, IL, USA) for Windows.

\section{RESULTS \\ Expression of Olfm Transcripts in the Non-Pregnant Fallopian Tubes}

The expression of Olfm-1, $-2,-3$ and -4 transcripts was studied in non-pregnant Fallopian tube. Tissues obtained from the follicular or luteal phases were dissected into three regions: infundibullary, ampullary and isthmic. The expression of Olfm-1 transcript was higher in the follicular phase than in the luteal phase in all three regions. A significantly higher $(P<0.05)$ expression of ampullary Olfm-1 in the follicular phase was observed (Figure 1a). Similarly, a significantly higher infundibullary Olfm-2 $(P<0.05)$ expression was found in the follicular phase than in the luteal phase. Interestingly, the expression of infundibullary Olfm-3 in the follicular phase was significantly higher $(P<0.05)$ than that 
a

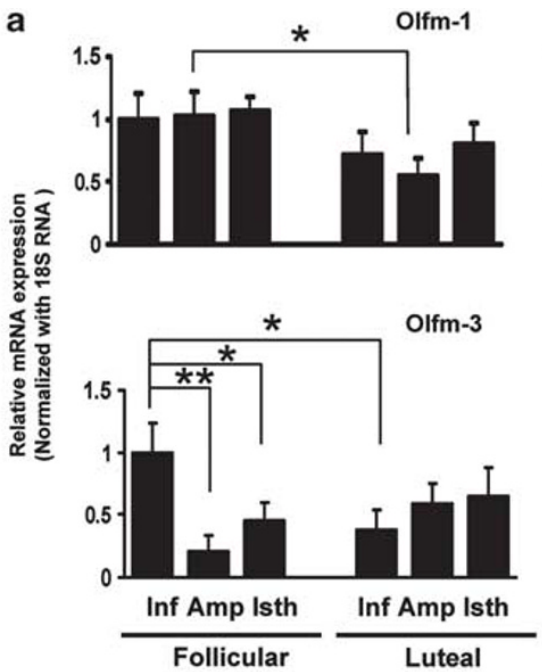

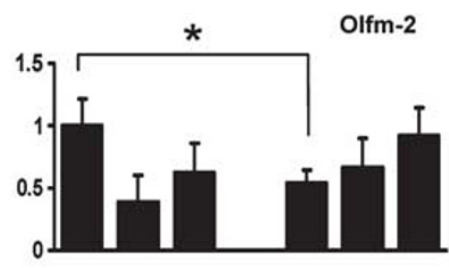

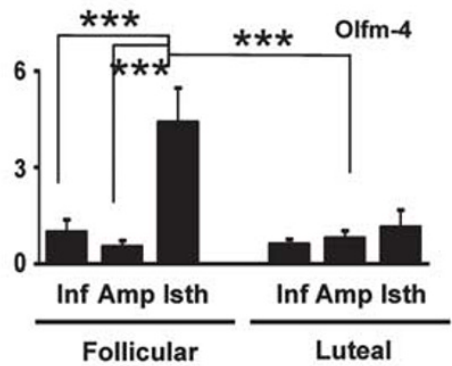

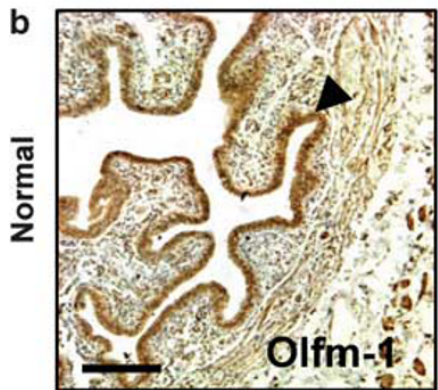
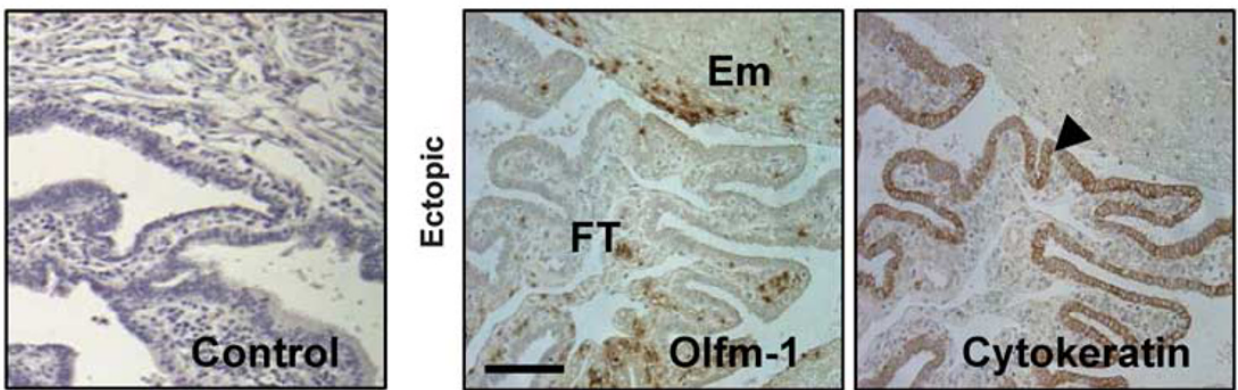

Figure 1 Olfactomedin (Olfm) transcripts are differentially expressed in non-pregnant Fallopian tube and Fallopian tube from women with ectopic pregnancy. (a) The expression of Olfm (Olfm-1, $-2,-3$ and -4) transcripts in different regions of non-pregnant Fallopian tube in the follicular ( $n=14$ ) and luteal $(n=15)$ phases of the menstrual cycle was quantified by real-time polymerase chain reaction (PCR). The expression of each Olfm transcript at the infundibulum region at the follicular phase of the cycle was an arbitrary set to a value of 1. Inf: infundibulum; Amp: ampulla; Isth: isthmus; ${ }^{\star} P<0.05$, ${ }^{* *} P<0.01$ and ${ }^{* * *} P<0.005$. (b) Olfm-1 protein is differentially expressed in the ampullary region of non-pregnant Fallopian tube at the luteal phase and Fallopian tube from women with ectopic pregnancy. The negative control section was included by using pre-absorbed antibody with blocking peptide (left panel). The arrows show the localization of Olfm-1 protein in the luminal epithelial cells of the ampullary region $(\times 400)$. The right panel illustrates the Olfm-1 and cytokeratin staining in the intact epithelium of the same TEP sample. FT: Fallopian tube; Em: embryonic tissues. Scale bar $=100 \mu$ m.

in the ampullary or isthmic regions in the follicular phase, as well as the infundibullary region in the luteal phase. Although the overall expression of Olfm-4 was very low in the Fallopian tube, there was a significant $(P<0.005)$ increase of its transcript in the isthmic region in the follicular phase when compared to that in the infundibullary and ampullary regions.

\section{Expression of Olfm-1 Protein in Non-Pregnant Fallopian Tubes and in Fallopian Tubes from Women with Ectopic Pregnancy}

Olfm-1 protein was mainly localized to the luminal epithelium and some scattered staining was found in the stroma of the Fallopian tube (Figure 1b, left panel). The expression of Olfm-1 protein in the Fallopian tube with TEP was reduced and localized to the stromal part of the Fallopian tube (Figure $1 \mathrm{~b}$, right panel). An intense cytokeratin 17 staining was observed in the ampullary epithelial cells, suggesting that the epithelium was intact in all of the ectopic sections studied. Table 1 summarizes the H-SCORE of Olfm-1 protein
Table 1 Olfactomedin-1 protein expression in non-pregnant normal and ectopic Fallopian tube

Fallopian tube region

H-SCORE (mean \pm s.d.)

\begin{tabular}{|c|c|c|}
\hline & $\begin{array}{l}\text { Follicular phase } \\
\qquad(n=8)\end{array}$ & $\begin{array}{l}\text { Luteal phase } \\
\qquad(n=10)\end{array}$ \\
\hline Infundibullary & $3.6 \pm 0.3^{\mathrm{a}}$ & $3.5 \pm 0.2^{\mathrm{a}}$ \\
\hline Ampullary & $2.5 \pm 0.3^{b}$ & $2.4 \pm 0.5^{b}$ \\
\hline Isthmic & $2.3 \pm 0.7^{b}$ & $2.3 \pm 0.6^{b}$ \\
\hline Ampullary TEP & \multicolumn{2}{|c|}{$1.3 \pm 0.4^{c}(n=10)$} \\
\hline
\end{tabular}

expression in non-pregnant Fallopian tubes and in Fallopian tubes from women with ectopic pregnancy. The expression of Olfm-1 protein was significantly higher $(P<0.05)$ in the 
infundibullary region than in the isthmic and ampullary regions in both the follicular (H-SCORE $=3.6 \pm 0.3$ vs $2.5 \pm 0.3$ vs $2.3 \pm 0.7$, respectively, $n=8$ ) and luteal (HSCORE $=3.5 \pm 0.2$ vs $2.4 \pm 0.5$ vs $2.3 \pm 0.6$, respectively, $n=10)$ phases of the cycle. The expression of Olfm-1 protein in the ampullary ectopic sections was significantly $(P<0.05)$ lower than that in the ampullae of non-pregnant Fallopian tube (H-SCORE $=1.3 \pm 0.4$ vs $2.4 \pm 0.5, n=10$; Table 1 ).

\section{Effect of Olfm-1 on JAr Spheroid Attachment onto OE-E6/E7 Cells}

The JAr cells were used to produce spheroids of sizes of about $60-200 \mu \mathrm{m}$ (Figure 2a) for the attachment studies. The spheroids attached to the OE-E6/E7 monolayer and the attachment rate increased with time. At 1,2 and $3 \mathrm{~h}$, the attachment rates were $25 \%, 48 \%$ and $65 \%$, respectively. Incubation of spheroids for $6 \mathrm{~h}$ or longer did not further increase the attachment rate (range: 65-75\%; Figure $2 \mathrm{~b}$ ). Therefore, a 4-h co-culture period was selected for the assay.
Interestingly, trophoblastic cell invasion like phenotype could be observed after prolonged $(>24 \mathrm{~h}$ ) co-culture (Figure 2a).

To test the effect of rhOlfm-1 on spheroid attachment onto OE-E6/E7 cells, we first detected the binding of rhOlfm-1 onto both cell types in vitro. It was found that AlexaFlour 488-labeled rhOlfm-1 bound to both the $\mathrm{JAr}^{16}$ and the OEE6/E7 cells (data not shown). Treatment of OE-E6/E7 cells with rhOlfm- 1 for $24 \mathrm{~h}$ dose-dependently decreased the JAr spheroid attachment onto the OE-E6/E7 monolayer (Figure 2c), and the reduction was significantly different $(P<0.01)$ from the untreated control. In addition, rhOlfm-1 at $5 \mu \mathrm{g} / \mathrm{ml}$ significantly $(P<0.05)$ suppressed attachment when compared with the $0.2 \mu \mathrm{g} / \mathrm{ml}$ group.

\section{Knockdown of Olfm-1 in OE-E6/E7 Cells Stimulates JAr Spheroid Attachment}

To determine whether the downregulation of Olfm-1 stimulated JAr spheroid attachment onto OE-E6/E7 cells, we used Olfm-1-specific siRNA for transfection study (Figure 2d). a
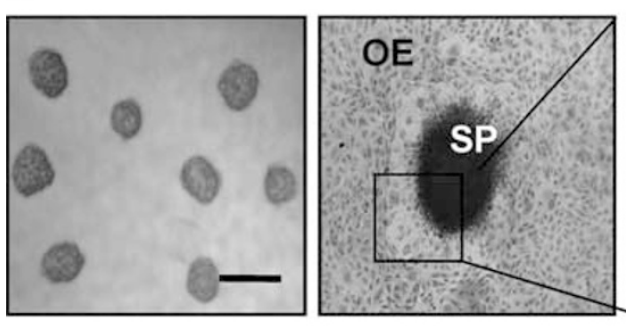

C

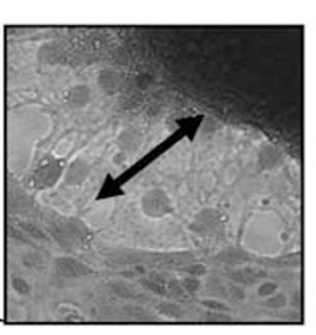

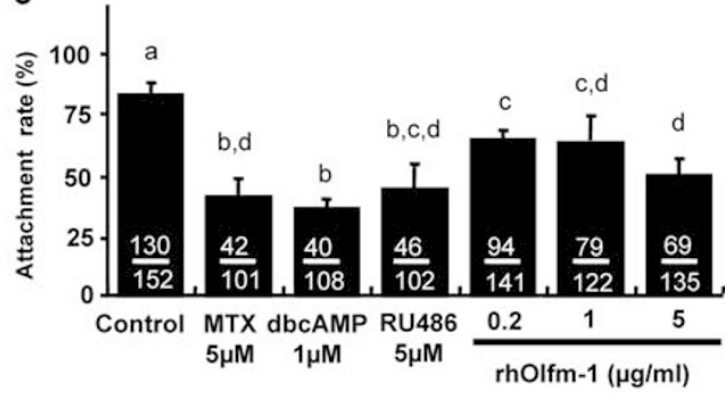

b

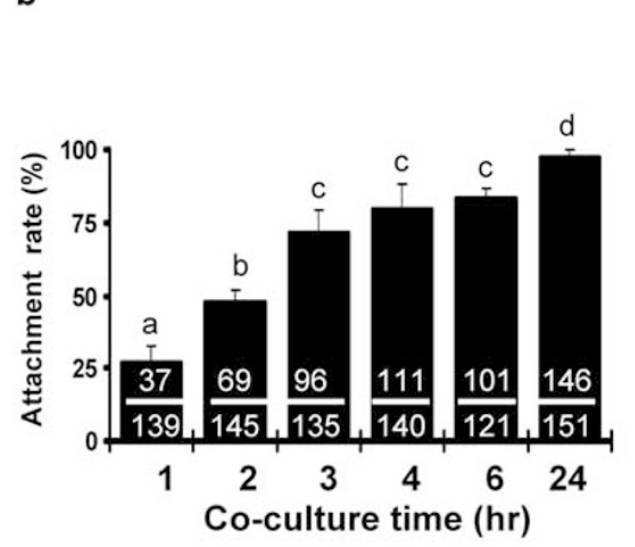

d
RNAi

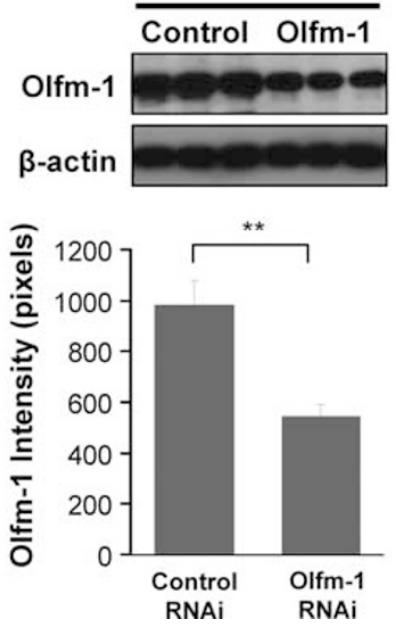

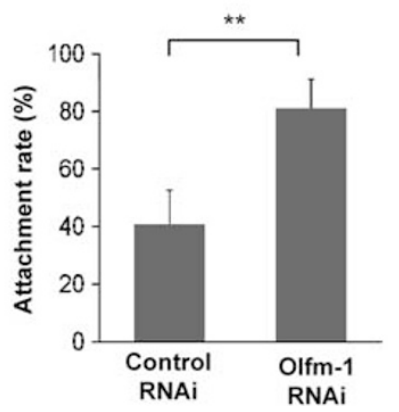

Figure 2 OE-E6/E7 monolayer and JAr trophoblastic spheroids are used in co-culture assay. (a) JAr spheroids of sizes 60-120 $\mu \mathrm{m}$ were selected (left) and co-cultured with an OE-E6/E7 monolayer (middle, OE) showing invasion of trophoblastic cells (right, double arrows). (b) The attachment rate of JAr spheroids onto OE-E6/E7 monolayer increased with time in the co-culture assay. (c) The effects of methotrexate (MTX), dibutyryl-cAMP (dbcAMP), RU486 and Olfm- 1 recombinant protein $(0.2,1$ and $5 \mu \mathrm{g} / \mathrm{ml})$ on attachment rate after $4 \mathrm{~h}$ co-culture were determined. ${ }^{\text {a-d }}$, Significant difference from each other at $P<0.05$. (d) Knockdown of Olfm-1 expression in OE-E6/E7 cells by small interfering RNA (siRNA) suppresses JAr spheroid attachment. Olfm-1 siRNA significantly downregulated Olfm-1 protein expression ( $>47 \%)$ in OE-E6/E7 monolayer when compared to the control siRNA (left panel). Knockdown of Olfm- 1 expression in OE-E6/E7 monolayer significantly increased ( $* * P<0.05$, twofold) JAr spheroid attachment onto OE-E6/E7 monolayer after $2 \mathrm{~h}$ co-culture when compared to the cells transfected with control RNA interference (right panel). 
Olfm-1 siRNA significantly $(P<0.05)$ downregulated $>45 \%$ of Olfm-1 expression when compared to OE-E6/E7 cells transfected with control siRNA. The JAr spheroid attachment rate onto the transfected OE-E6/E7 monolayer was significantly increased $(P<0.001)$ by twofold $(39 \%$ vs $82 \%)$ after co-culture for $2 \mathrm{~h}$ when compared to the control (control siRNA; Figure 2d).

\section{Wnt Activation Suppresses Olfm-1 Expression and Increases JAr Spheroid Attachment onto OE-E6/E7 Cells} To determine whether activation of the Wnt-signaling pathway could restore Olfm- 1 suppression and spheroid attachment, we used Wnt3a-CM (1:1 ratio) and $\mathrm{LiCl}(40 \mathrm{mM})$ to treat the OE-E6/E7 cells for $24 \mathrm{~h}$ and studied the changes in the expression of Wnt-signaling molecules and spheroid attachment rates (Figure 3a). Both $\mathrm{Wnt} 3 \mathrm{a}-\mathrm{CM}$ and $\mathrm{LiCl}$ activated Wnt signaling as demonstrated by the upregulation of $\beta$-catenin expression in the treated OE-E6/E7 cells. Interestingly, the treatments were associated with a significant decrease in Olfm-1 protein expression at 1:1 ratio of Wnt3a-CM:DMEM/F12 and $40 \mathrm{mM}$ of $\mathrm{LiCl}$ (Figure 3a). Treatment of OE-E6/E7 cells with either Wnt3a-CM or LiCl stimulated spheroid attachment significantly when compared to the controls (Figure 3b). Wnt3a-CM and LiCl significantly $(P<0.001)$ increased (three- and twofold, respectively) spheroid attachment rates after co-culture with OE-E6/E7 for $1 \mathrm{~h}$. Addition of the Wnt-inhibitor Dkk-1 $(0.1 \mu \mathrm{g} / \mathrm{ml})$ or rhOlfm-1 $(0.2 \mu \mathrm{g} / \mathrm{ml})$ to the OE-E6/E7 cells for $24 \mathrm{~h}$ nullified the stimulatory effect of Wnt3a-CM or LiCl on JAr spheroid attachment (Figure 3b).

\section{DISCUSSION}

This study provides fundamental data on the spatiotemporal expression and regulation of Olfm-1 in the human Fallopian tube and is the first report of the association of activation of Wnt signaling and suppression of Olfm-1 in ectopic pregnancy. We demonstrate that Olfm-1 expression is reduced in the epithelium of the Fallopian tube of women with ectopic pregnancy compared to Fallopian tube from non-pregnant women. In addition, our novel in vitro model of tubalembryo attachment demonstrates that Olfm-1 suppresses trophoblastic spheroid attachment onto Fallopian tubal epithelial cells, whereas Wnt-signaling activation, or RNAi knockdown of Olfm-1, increases the attachment.

In the non-pregnant human Fallopian tube, the expression of the Olfm-1 to -4 mRNAs were significantly higher in the follicular phase than in the luteal phase of the menstrual cycle, suggesting that their expression might be hormonally regulated. Similarly, Olfm-1 mRNA is also downregulated in the secretory phase of the human endometrium. ${ }^{16}$ Interestingly, the expression of ampullary Olfm-1 transcript was significantly lower $(P<0.05)$ at the luteal phase when compared to the follicular phase, although the expression of Olfm-1 protein was similar in the same region at both phases of the cycle. The discrepancy would be due to changes in mRNA stability and protein turnover in response to different a
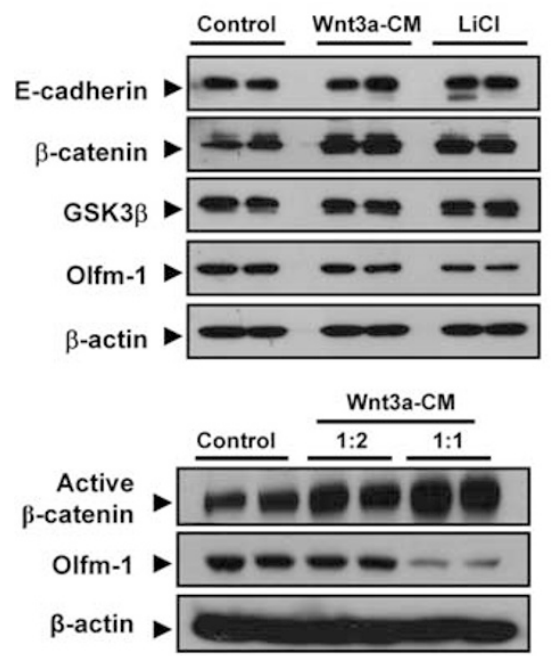

b
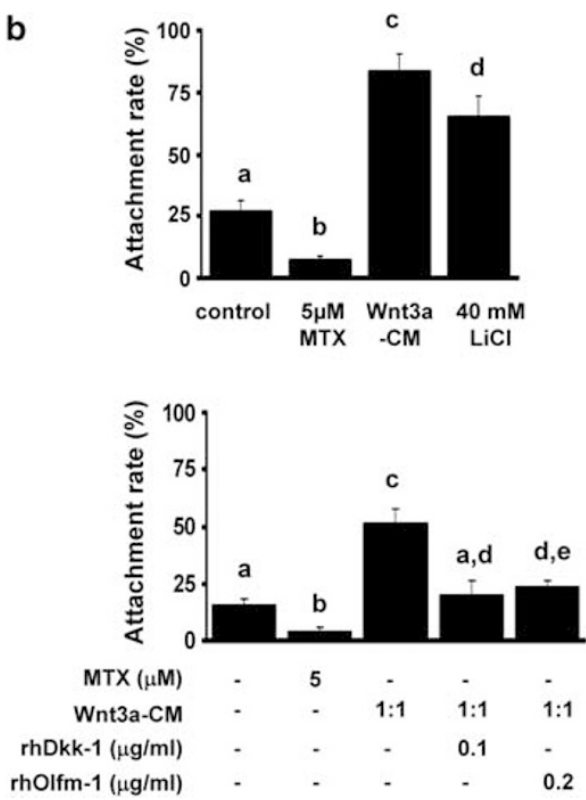

Figure 3 Activation of Wnt signaling downregulates Olfm-1 expression and reverses the suppressive effect of Olfm-1 on spheroid attachment. (a, upper panel) The activation of Wnt-signaling pathway by Wnt3a-conditioned medium (1:2 ratio) or LiCl ( $40 \mathrm{mM}$ ) resulted in an elevated total $\beta$-catenin, but reduced Olfm-1 expression. No change in the expression of GSK-3 $\beta$ and $\beta$-actin was found. The lower panel depicts the changes of active- $\beta$-catenin and Olfm-1 in the OE-E6/E7 cells treated $24 \mathrm{~h}$ with Wnt3a-CM. (b) Wnt3a-CM and LiCl stimulate JAr spheroid attachment onto OE-E6/E7 monolayer after $1 \mathrm{~h}$ co-culture (upper panel). The increased JAr spheroid attachment by Wnt3a-CM can be nullified by co-treatment of Wnt3a-CM with rhOlfm-1 or rhDkk-1 (lower graph). ${ }^{\mathrm{a}-\mathrm{e}}$, Significant difference from each other at $P<0.05$. 
hormonal regulation. More than $70 \%$ of ectopic pregnancies are found in the ampullary region of the Fallopian tube, whereas implantation in the infundibullary or isthmic portions is rare $(<5 \%){ }^{29}$ The higher expression of Olfm-1 protein in the infundibullary region than in the ampullary and isthmic regions in non-pregnant human Fallopian tube supports the low percentage of TEP found in the infundibulum region of the Fallopian tube. ${ }^{1}$

Bioinformatics was used in the analysis of the promoter region of the OLFM1 gene and no consensus estrogen-responsive element (ERE) or progesterone-responsive element (PRE) was identified. Therefore, it is unlikely that ovarian steroids regulate Olfm-1 expression in the Fallopian tube epithelium through ERE or PRE binding. Interestingly, a number of ITF- 2 binding sites were identified at the Olfm-1 promoter region. ITF- 2 expression is regulated by $\mathrm{Wnt} / \beta$-catenin signaling pathway. ${ }^{30}$ Our data also showed that Wnt activation downregulated Olfm-1 expression in OE-E6/E7 cells and increased the JAr spheroid attachment in vitro. Further studies on Olfm-1 promoter will help to understand the regulatory mechanism of Olfm-1 expression and also to delineate the interaction of Olfm-1 and Wnt signaling in the Fallopian tube.

Olfm protein was significantly downregulated in the Fallopian tube of patients with ectopic pregnancy when compared to the non-pregnant patients. However, it was noted that patients in the non-pregnant group is older than the ectopic pregnancy group. Moreover, the differences in Olfm-1 expression could be due to the increase in steroid hormone levels in patients with ectopic pregnancy (7-10 weeks gestational period). Therefore, comparison between patients with ectopic pregnancy and normal pregnancy at similar gestational period warrants further investigations. However, the collection of Fallopian tube in normal pregnant women at similar gestational period is practically difficult. Despite this, those pregnant women who seek termination of pregnancy and tubal ligation could be recruited.

To our knowledge, this is the first report of an in vitro model of tubal pregnancy. There are numerous studies using co-culture of human embryos and endometrium for the study of endometrial biology. ${ }^{31}$ However, similar studies using Fallopian tube, designed for further understanding of the etiology of ectopic pregnancy are lacking owing to limited availability of cell lines. Our OE-E6/E7 cells retain most of the Fallopian tube epithelial cell characters, including morphological, hormonal responses and expression of receptor, ${ }^{24,32,33}$ and many early implantation-related molecules, including Olfm- $1, \beta$-catenin, E-cadherin, LIF, intergrin $\alpha \mathrm{V}$ and $\beta 3$, MUC1, ERK and JNK (data not shown). The trophoblastic JAr cell line was used as embryo surrogate based on its expression of many cytotrophoblastic markers. ${ }^{34,35}$ The OE-E6/E7 and JAr cell lines also provide flexibility for gene manipulation (eg, siRNA or plasmid DNA) in functional studies. We believe that this co-culture model could be used to study the functional role of other proteins in embryo attachment and ectopic pregnancy.
Since Olfm-1 is an integral part of the ECM, downregulation of Olfm-1 may be associated with changes in the expression of other ECM molecules, thereby affecting cell-cell interaction. A number of other ECM molecules, including integrins, ${ }^{36}$ mucin $1,{ }^{37,38}$ cadherins, ${ }^{39}$ laminins and fibronectins,${ }^{36}$ have been associated with TEP. For example, a reduced expression of the MUC1 mucin has been noted in the Fallopian tube from women with ectopic pregnancies. ${ }^{38,40}$ MUC1 is proposed to function as a barrier for embryo implantation in the endometrium. ${ }^{41-45}$ Knockdown of Olfm-1 expression with siRNA in the OE-E6/E7 cells increased trophoblast spheroid attachment in the present in vitro model, supporting our hypothesis that Olfm-1 may also function as a barrier for embryo attachment. It is possible that downregulation of Olfm-1 and MUC1 expression ${ }^{38}$ contribute synergistically for ectopic pregnancy.

Activation of the canonical Wnt-signaling pathway by Wnt3a or LiCl downregulates Olfm-1 expression in the OEE6/E7 cells and significantly stimulates JAr spheroid attachment. It is possible that the reduced Olfm-1 expression also allows exposure of other adhesion molecules for embryo attachment. Previous studies have shown that activation of the canonical Wnt-signaling molecule $\beta$-catenin favors adhesion junction formation through a cadherin-catenin complex. ${ }^{46}$ In line with this, the implanting embryo secretes canonical Wntsignaling activators (eg, Wnt3a) to regulate the expression of genes responsible for ectopic implantation. ${ }^{47,48}$ Yet, how activation of canonical Wnt-signaling affects gene expression leading to ectopic pregnancy warrants further investigations.

It is generally believed that pelvic infection triggers an immune response and stimulates secretion of various cytokines, including interleukins, ${ }^{49} \mathrm{TNF}^{5},{ }^{50}$ TGF- $\beta$ and interferons that are associated with ectopic pregnancy. ${ }^{51}$ Interestingly, TNF $\alpha$ and TGF- $\beta$ downregulate Olfm- 1 mRNA expression in the podocytes. ${ }^{52}$ Whether pelvic infection changes in cytokine expression act synergistically with Olfm-1 in the Fallopian tube leading to ectopic pregnancy remains to be investigated.

In summary, our data show that Olfm-1 is differentially expressed in the human Fallopian tube and activation of the Wnt-signaling pathway or suppression of Olfm-1 expression in the Fallopian tubal cells increases spheroid attachment. A better understanding of the role of adhesion-related molecules (eg, Olfm and MUC1) in the human Fallopian tube may help to delineate the underlying mechanism leading to ectopic pregnancies and better prevention of tubal ectopic pregnancies in the future.

Supplementary Information accompanies the paper on the Laboratory Investigation website (http://www.laboratoryinvestigation.org)

\section{ACKNOWLEDGEMENTS}

This work was supported in part by grants from the Committee on Research and Conference Grant, The University of Hong Kong to KFL and Hong Kong Research Grant Council. AWH is supported by an MRC Clinician Scientist Fellowship. 


\section{DISCLOSURE/CONFLICT OF INTEREST}

The authors declare no conflict of interest.

1. DeCherney $\mathrm{AH}$, Boyers SP. Isthmic ectopic pregnancy: segmenta resection as the treatment of choice. Fertil Steril 1985;44:307-312.

2. Shaw JL, Dey SK, Critchley HO, et al. Current knowledge of the aetiology of human tubal ectopic pregnancy. Hum Reprod Update 2010;16:432-444.

3. Snyder DA, Rivers AM, Yokoe $H$, et al. Olfactomedin: purification, characterization, and localization of a novel olfactory glycoprotein Biochemistry 1991;30:9143-9153.

4. Tomarev SI, Nakaya N. Olfactomedin domain-containing proteins: possible mechanisms of action and functions in normal development and pathology. Mol Neurobiol 2009;40:122-138.

5. Danielson PE, Forss-Petter S, Battenberg EL, et al. Four structurally distinct neuron-specific olfactomedin-related glycoproteins produced by differential promoter utilization and alternative mRNA splicing from a single gene. J Neurosci Res 1994;38:468-478.

6. Goldwich A, Scholz M, Tamm ER. Myocilin promotes substrate adhesion, spreading and formation of focal contacts in podocytes and mesangial cells. Histochem Cell Biol 2009;131:167-180.

7. Harland RM. A protein scaffold plays matchmaker for chordin. Cell 2008;134:718-719.

8. Inomata H, Haraguchi T, Sasai Y. Robust stability of the embryonic axial pattern requires a secreted scaffold for chordin degradation. Cell 2008;134:854-865.

9. Nakaya N, Lee HS, Takada $\mathrm{Y}$, et al. Zebrafish olfactomedin 1 regulates retinal axon elongation in vivo and is a modulator of Wnt signaling pathway. J Neurosci 2008;28:7900-7910.

10. Borthwick JM, Charnock-Jones DS, Tom BD, et al. Determination of the transcript profile of human endometrium. Mol Hum Reprod 2003:9:19-33.

11. Horcajadas JA, Riesewijk A, Martin J, et al. Global gene expression profiling of human endometrial receptivity. J Reprod Immunol 2004;63:41-49.

12. Liu $\mathrm{Y}$, Lee $\mathrm{KF}, \mathrm{Ng} \mathrm{EH}$, et al. Gene expression profiling of human periimplantation endometria between natural and stimulated cycles. Fertil Steril 2008;90:2152-2164.

13. Riesewijk A, Martin J, van Os R, et al. Gene expression profiling of human endometrial receptivity on days $\mathrm{LH}+2$ vs $\mathrm{LH}+7$ by microarray technology. Mol Hum Reprod 2003;9:253-264.

14. Kao LC, Germeyer A, Tulac S, et al. Expression profiling of endometrium from women with endometriosis reveals candidate genes for diseasebased implantation failure and infertility. Endocrinology 2003;144: 2870-2881.

15. Lee J, Oh J, Choi E, et al. Differentially expressed genes implicated in unexplained recurrent spontaneous abortion. Int J Biochem Cell Biol 2007;39:2265-2277

16. Kodithuwakku SP, Ng PY, Liu $\mathrm{Y}$, et al. Hormonal regulation of endometrial olfactomedin expression and its suppressive effect on spheroid attachment onto endometrial epithelial cells. Hum Reprod 2011;26:167-175.

17. Funayama $T$, Mashima $Y$, Ohtake $Y$, et al. SNPs and interaction analyses of noelin 2, myocilin, and optineurin genes in Japanese patients with open-angle glaucoma. Invest Ophthalmol Vis Sci 2006:47:5368-5375.

18. Mohamed OA, Dufort D, Clarke HJ. Expression and estradiol regulation of Wnt genes in the mouse blastocyst identify a candidate pathway for embryo-maternal signaling at implantation. Biol Reprod 2004;71:417-424.

19. Mohamed OA, Jonnaert M, Labelle-Dumais C, et al. Uterine Wnt/betacatenin signaling is required for implantation. Proc Natl Acad Sci USA 2005;102:8579-8584.

20. Yeung WS, Ng VK, Lau EY, et al. Human oviductal cells and their conditioned medium maintain the motility and hyperactivation of human spermatozoa in vitro. Hum Reprod 1994;9:656-660.

21. Livak KJ, Schmittgen TD. Analysis of relative gene expression data using real-time quantitative PCR and the 2(-Delta Delta C(T)) Method. Methods 2001;25:402-408.

22. Wei Q, St Clair JB, Fu T, et al. Reduced expression of biomarkers associated with the implantation window in women with endometriosis. Fertil Steril 2009;91:1686-1691.
23. Liu Y, Kodithuwakku SP, Ng PY, et al. Excessive ovarian stimulation upregulates the Wnt-signaling molecule DKK1 in human endometrium and may affect implantation: an in vitro co-culture study. Hum Reprod 2010;25:479-490.

24. Lee $\mathrm{YL}$, Lee $\mathrm{KF}, \mathrm{Xu}$ JS, et al. Establishment and characterization of an immortalized human oviductal cell line. Mol Reprod Dev 2001;59:400-409.

25. Aboussahoud W, Bruce C, Elliott S, et al. Activation of Toll-like receptor 5 decreases the attachment of human trophoblast cells to endometrial cells in vitro. Hum Reprod 2010;25:2217-2228.

26. Heneweer C, Kruse LH, Kindhauser F, et al. Adhesiveness of human uterine epithelial RL95-2 cells to trophoblast: rho protein regulation. Mol Hum Reprod 2002;8:1014-1022.

27. Kishida S, Yamamoto H, Kikuchi A. Wnt-3a and Dvl induce neurite retraction by activating Rho-associated kinase. Mol Cell Biol 2004;24:4487-4501.

28. Willert K, Brown JD, Danenberg $\mathrm{E}$, et al. Wnt proteins are lipid-modified and can act as stem cell growth factors. Nature 2003;423:448-452.

29. Bouyer J, Coste J, Fernandez H, et al. Sites of ectopic pregnancy: a 10 year population-based study of 1800 cases. Hum Reprod 2002;17: 3224-3230.

30. Kolligs FT, Nieman MT, Winer I, et al. ITF-2, a downstream target of the Wnt/TCF pathway, is activated in human cancers with beta-catenin defects and promotes neoplastic transformation. Cancer Cell 2002; 1:145-155.

31. Teklenburg G, Macklon NS. Review: in vitro models for the study of early human embryo-endometrium interactions. Reprod Sci 2009;16:811-818.

32. Aboussahoud W, Aflatoonian R, Bruce $C$, et al. Expression and function of Toll-like receptors in human endometrial epithelial cell lines. J Reprod Immunol 2010;84:41-51.

33. Monkkonen KS, Aflatoonian R, Lee KF, et al. Hormonal regulation of Galphai2 and mPRalpha in immortalized human oviductal cell line OEE6/E7. Mol Hum Reprod 2007;13:845-851.

34. Hannan NJ, Paiva P, Dimitriadis E, et al. Models for study of human embryo implantation: choice of cell lines? Biol Reprod 2010;82:235-245.

35. Apps R, Murphy SP, Fernando R, et al. Human leucocyte antigen (HLA) expression of primary trophoblast cells and placental cell lines, determined using single antigen beads to characterize allotype specificities of anti-HLA antibodies. Immunology 2009;127: 26-39.

36. Qin L, Wang YL, Bai SX, et al. Expression of integrins and extracellular matrix proteins at the maternal-fetal interface during tubal implantation. Reproduction 2003;126:383-391.

37. Al-Azemi M, Refaat B, Amer $\mathrm{S}$, et al. The expression of inducible nitric oxide synthase in the human fallopian tube during the menstrual cycle and in ectopic pregnancy. Fertil Steril 2010;94:833-840.

38. Al-Azemi M, Refaat B, Aplin J, et al. The expression of MUC1 in human Fallopian tube during the menstrual cycle and in ectopic pregnancy. Hum Reprod 2009;24:2582-2587.

39. Revel A, Ophir I, Koler $M$, et al. Changing etiology of tubal pregnancy following IVF. Hum Reprod 2008;23:1372-1376.

40. Savaris RF, da Silva LC, Moraes Gda S, et al. Expression of MUC1 in tubal pregnancy. Fertil Steril 2008;89:1015-1017.

41. Carson DD, DeSouza MM, Kardon $\mathrm{R}$, et al. Mucin expression and function in the female reproductive tract. Hum Reprod Update 1998;4:459-464.

42. Carson DD, Julian J, Lessey BA, et al. MUC1 is a scaffold for selectin ligands in the human uterus. Front Biosci 2006;11:2903-2908.

43. DeSouza MM, Surveyor GA, Price RE, et al. MUC1/episialin: a critical barrier in the female reproductive tract. J Reprod Immunol 1999; 45:127-158.

44. Hey NA, Graham RA, Seif MW, et al. The polymorphic epithelial mucin MUC1 in human endometrium is regulated with maximal expression in the implantation phase. J Clin Endocrinol Metab 1994;78:337-342.

45. Hey NA, Meseguer M, Simon C, et al. Transmembrane and truncated (SEC) isoforms of MUC1 in the human endometrium and Fallopian tube. Reprod Biol Endocrinol 2003;1:2.

46. Gooding JM, Yap KL, Ikura M. The cadherin-catenin complex as a focal point of cell adhesion and signalling: new insights from threedimensional structures. Bioessays 2004;26:497-511. 
47. Hierholzer A, Kemler R. Beta-catenin-mediated signaling and cell adhesion in postgastrulation mouse embryos. Dev Dyn 2010;239:191-199.

48. Kemp C, Willems E, Abdo S, et al. Expression of all Wnt genes and their secreted antagonists during mouse blastocyst and postimplantation development. Dev Dyn 2005;233:1064-1075.

49. Iyibozkurt AC, Kalelioglu I, Gursoy S, et al. Evaluation of serum levels of interleukin-10, interleukin-11 and leukemia inhibitory factor in differentiation of eutopic and tubal ectopic pregnancies. Clin Exp Obstet Gynecol 2010;37:217-220.
50. Laskarin G, Redzovic A, Vukelic P, et al. Phenotype of NK cells and cytotoxic/apoptotic mediators expression in ectopic pregnancy. Am J Reprod Immunol 2010;64:347-358.

51. Tonello A, Poli G. Tubal ectopic pregnancy: macrophages under the microscope. Hum Reprod 2007;22:2577-2584.

52. Bohr DC, Koch $M$, Kritzenberger $M$, et al. Increased expression of olfactomedin-1 and myocilin in podocytes during puromycin aminonucleoside nephrosis. Nephrol Dial Transplant 2010;26:83-92. 